\title{
THE IMPACT OF BALANCE AND VISUAL FEEDBACK ON TIBIALIS ANTERIOR/GASTROCNEMIUS NEUROMUSCULAR ACTIVATION TIMING IN HEALTHY YOUNG ADULTS
}

original paper

(1) University School of Physical Education in Wroclaw

DOI: https://doi.org/10.5114/hm.2021.103289

\section{MARTÍN G. ROSARIO, ALEENA JOSE}

Physical Therapy Program, Texas Woman’s University, Dallas, USA

\begin{abstract}
Purpose. Previous studies have compared neuromuscular patterns between healthy populations and populations with neuromuscular disorders where the functionality of the muscles might be affected. However, more research needs to be conducted to understand neuromuscular patterns within young healthy adults during various balance tasks. This study explores whether challenging the sensory systems in young adults during single and dual-cognitive balance tasks alters neuromuscular patterns at the gastrocnemius and tibialis anterior.

Methods. Our study involved 44 healthy young adults (10 males and 34 females) with an average age of $24.9 \pm 3.03$ years. Electromyography electrodes were utilized to obtain electromyographic data regarding times during onset of muscle contraction, peak muscle activation, decay of muscle contraction, and duration of muscle contraction during distinct balance tests. The different balance tests involved altering sensory inputs during 4 single tasks and 4 dual-cognitive tasks.

Results. Principally, no significant changes in electromyographic activity were demonstrated during the single or dualcognitive tests, especially for neuromuscular recruitment patterns for the tibialis anterior and gastrocnemius $(p>0.05)$.

Conclusions. The comparable neuromuscular activity of the tibialis anterior and gastrocnemius during various balance tasks in our study extrapolates that young healthy adults have functional sensory and motor systems. Further studies need to investigate electromyographic patterns during dual-motor tasks, the involvement of other synergistic muscles for postural control, and brain activity during complex postural tasks.
\end{abstract}

Key words: electromyography, standing balance, lower limb musculature

\section{Introduction}

The ability to maintain an upright stance requires substantial interaction between the sensory systems (afferent input), sensory processing at the central nervous system, and the neuromuscular systems. The various sensory systems (visual, somatosensory, vestibular) gather information or input to understand the body's position in space; sensory processing allows integration of the sensory input from the sensory systems, and then a musculoskeletal (efferent) response such as activation of postural muscles is initiated to maintain balance [1]. The integration of the different systems enables postural stability by ensuring that the centre of mass is over the base of support [1]. In humans, the base of support refers to the area beneath in which the body or object contacts the ground, which could include arms, legs, or a chair [2].
Neuromuscular disorders refer to any diseases that affect the sensory and/or motor nerves in our body, which ultimately can lead to inefficient muscular responses required for postural stability. Various neuromuscular disorders can also impede balance strategies following the deterioration of neuronal synapses or inconsistent release of chemicals at the neuromuscular junction, potentially hindering or altering the activation of muscles responsible for balance control. The neuromuscular junction is where the axons carrying the neural signals from the spinal cord or brainstem elicit a motor response at the muscle fibres they innervate [3]. Illustrations of these disturbances include amyotrophic lateral sclerosis, multiple sclerosis, myasthenia gravis, peripheral neuropathy, and postpolio syndrome [4]. Diabetes mellitus is another form of neuromuscular disorder affecting balance which may be accompanied by polyneuropathy and retinop-

Correspondence address: Martín G. Rosario, Physical Therapy Program, Texas Woman’s University, Dallas Campus; 5500 Southwestern Medical Ave., Dallas, TX 75235-7299, USA, e-mail: mrosario1@twu.edu

Received: June 1, 2020

Accepted for publication: July 6, 2020

Citation: Rosario MG, Jose A. The impact of balance and visual feedback on tibialis anterior/gastrocnemius neuromuscular activation timing in healthy young adults. Hum Mov. 2021;22(4):46-55; doi: https://doi.org/10.5114/hm.2021.103289. 
athy that conjunctively affects the proprioceptive and visual systems. Other disorders that affect the sensory and cognitive systems can also entail postural disturbances. Psychiatric disorders (anxiety and depression) and cognitive disorders (dementia) can also manifest vestibular symptoms and distinct balance alterations [5]. Additionally, sensory processing disorder is a diagnosis that often involves abnormal hyperactive or hypoactive responses to sensory input, though this diagnosis is usually seen in children with developmental disabilities [6]. Like in children with autism spectrum disorder, these disparities in sensory processing might be the explanation for the increased standing postural sway [7].

Employing balance measurement tools and tests enables clinicians and researchers to identify the underlying pathology behind balance impairments, as well as keep track of advancements on improvements after balance training. Numerous qualitative measures have been established to assess balance (Activities-specific Balance Confidence scale, Balance Error Scoring System test, Berg Balance Scale) in the neuromuscular populations. Utilizing technological equipment, for instance, motion sensors, can propose an objective measure of balance by analysing the displacement of the centre of pressure and capturing balance distinctions that are insensitive to qualitative measures. Centre of pressure is defined as a point in which the weight of the body over the foot would be concentrated [1]. Such devices can be employed in athletes or individuals with intact sensory systems or minimal alteration that otherwise would show a ceiling effect if qualitative balance assessments were utilized. Additionally, electromyography (EMG) electrode systems capture muscle activation (electrical activity) as a numerical value that can further depict muscle response during balance activities.

Postural stability is attained through a dynamic interaction among the postural muscles at the trunk, hip, knee, and ankle. Earlier studies have contended that muscle weakness can cause postural instability and falls owing to decreased joint stabilization, and the coordination of ankle muscles primarily plays an essential role in balance recovery $[8,9]$. Thence, it is critical to consider muscle strength prior to assessing balance.

Prior research has extensively explored abnormal variations in EMG activity in the neuromuscular populations, like longer latency in EMG postural response in those with multiple sclerosis and neuropathy [10]. There is relatively limited evidence regarding neuromuscular response and coordination during dual tasks whilst challenging the sensory systems in young adults.
One study found increased dorsiflexion activation during dual task compared with single task [11]. A study by Little and Woollacott [12] also identified interference in central processing and changes in event-related potentials when posture was perturbed in young adults. Previous literature has recognized alterations in postural response that are more prominent in the neuromuscular populations compared with healthy adults. Rather than comparing our participants with neuromuscular populations, our study aims to identify whether complex balance tasks can impose postural imbalances within young healthy adults.

This study attempts to answer the following: can single and dual-cognitive balance activities in combination with different challenging scenarios provoke a distinct neuromuscular pattern in lower limb musculature in young healthy adults? The current study investigates the neuromuscular timing activity of the ankle muscles (tibialis anterior [TA] and gastrocnemius [GA]) while simultaneously challenging the sensory systems during single and dual-cognitive tasks in young adults. We hypothesized that the neuromuscular timing activity of TA and GA would be indistinguishable under the different sensory conditions owing to the presumptive intact sensory systems and efferent motor response in young adults.

\section{Material and methods}

\section{Participants}

The participants were recruited via word of mouth from research assistants at the Texas Woman's University Dallas campus. After the research assistants gave an overview of the study tasks, each participant signed the informed consent upon agreement prior to a subjective screening. The screening time was allotted to collect demographic information, including weight, age, height, hand/foot dominance, and grip strength by using a Camry EH101 electronic hand-held dynamometer.

In accordance with the inclusion criteria, the participants were to be young healthy adults within the age range of $18-45$ years to avoid any age-related variations to balance control.

The exclusion was established to avoid any external factors that could influence muscular response. The exclusion criteria were as follows: (1) pregnancy or conviction about pregnancy; (2) inability to maintain postural control for 30 seconds during the Romberg test, indicating balance problems; (3) BMI $>40 \mathrm{~kg} / \mathrm{m}^{2}$ or morbid obesity; (4) hypertension; (5) any surgeries 
or lesions at the back or lower extremity within the previous 6 months; (6) any untreated visual acuity deficits or difficulty reading prints on medication bottles even when wearing prescription glasses; (7) any use of drugs that cause drowsiness persisting more than 24 hours prior to balance testing.

Table 1 illustrates the participants' demographic profile. A total of 44 participants (10 males and $34 \mathrm{fe}$ males with an average age of $24.9 \pm 3.03$ years) completed this study. Additionally, to establish the leg used for EMG electrode placement, the dominant leg of each subject was determined. Of the 44 participants, 2 were left-leg dominant, and the remaining 42 were rightleg dominant. The dominant arm of each subject was also determined and used for grip strength testing. Of the 44 participants, 2 were left-hand dominant, and the remaining 42 were right-hand dominant. All data collection occurred at Texas Woman's University, T. Boone Pickens Institute of Health Sciences.

Table 1. Demographic data of all participants

\begin{tabular}{lc}
\hline Characteristics & Study participants $(n=44)$ \\
\hline Age (years) & $24.9 \pm 3.03$ \\
Gender & males: 10, females: 34 \\
Height (inches) & $66.4 \pm 3.8$ \\
Weight (pounds) & $148.0 \pm 23.3$ \\
Body mass index $\left(\mathrm{kg} / \mathrm{m}^{2}\right)$ & $23.6 \pm 1.4$ \\
\hline
\end{tabular}

\section{Balance and EMG assessment}

The EMG surface electrode system (Delsys, Inc., Boston, USA) was utilized to collect electrical activity of TA and GA at $1000 \mathrm{~Hz}$. The EMG electrode place- ment was based on the recommendations by Sacco and Kasman (Figure 1). First, the participants performed 2 trials of grip strength with the dominant hand. An EMG surface electrode was then placed on the dominant leg over the TA and GA. A non-electric razor was used to remove any hair at the electrode placement site that could hamper EMG data transmitted to the EMG electrodes.

A baseline measure of EMG activity was obtained by asking the participant to maintain a static bipedal stance on a firm surface while concurrently gazing at an orange square on a wall 10-feet away. The remaining 8 tests also required the aforementioned stance and gazing at the square, but were performed on a foam pad (12.5 inches wide, 15.5 inches long, 2.4 inches high) placed on a firm surface. The 8 balance tests consisted of 4 single tasks and 4 dual-cognitive tasks (counting backwards from 100 by threes). EMG activity was recorded for 15 seconds for each balance test from TA and GA simultaneously. For the balance tests requiring head movements, the participants performed head nods in the vertical direction following a metronome set at a cadence of $60 \mathrm{bpm}$. The single tasks were performed before the dual tasks.

The 4 single tasks on foam were as follows: (1) eyes open (EO); (2) eyes closed (EC); (3) eyes open with head nods (EOHUD); (4) eyes closed with head nods (ECHUD).

The 4 cognitive tasks on foam were as follows: (1) eyes open during cognitive task (EOC); (2) eyes closed during cognitive task (ECC); (3) eyes open with head nods during cognitive task (EOCHUD); (4) eyes closed with head nods during cognitive task (ECCHUD).

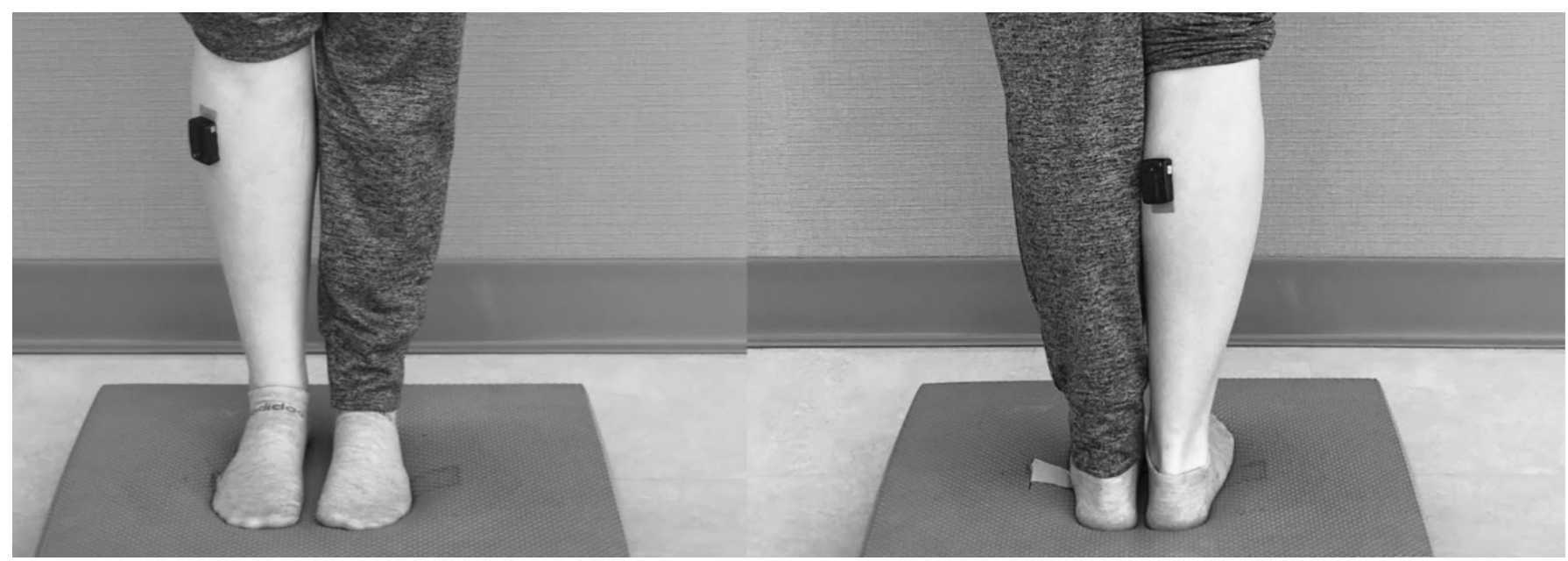

Tibialis anterior

Gastrocnemius

Figure 1. EMG electrode placement 


\section{Data analysis}

EMG data were captured by EMG works and processed by EMG analysis for both the TA and GA muscles. The investigators distinguished 3 activation points during the EMG trace for each muscle during all trials. The variable of interest in this endeavour is the time to the onset of muscle activation (onset). Furthermore, the investigation collected time to maximal peak activation (TP), decay or relaxation of maximal peak activation (decay), and duration of muscle activation (duration). The EMG data collection involved an average of 3 consecutive activation points for each muscle during all trials and tasks. For the data analysis, we utilized the SPSS software (version 25) with a MANOVA for the comparison within TA during single and dual tasks, and GA during single and dual tasks. Additionally, to understand the neuromuscular coordination and modification, we applied a MANOVA analysis and compared TA vs. GA during single and dual tasks. Finally, the current study performed a repeated measure ANOVA to compare the means for all neuromuscular-time variables across all tasks. This project examined the impact of single and dual-cognitive balance on all variables of interest (onset, TP, decay, and duration) by comparing these variables and the 2 muscles' timing within tasks. To adapt to the repeated measure ANOVA and MANOVA, we considered statistical significance of the results at $p<0.01$.

\section{Ethical approval}

The research related to human use has complied with all the relevant national regulations and institutional policies, has followed the tenets of the Declaration of Helsinki, and has been approved by the institutional review board of Texas Woman's University (protocol \# 20092).

\section{Informed consent}

Informed consent has been obtained from all individuals included in this study.

\section{Results}

Table 2 shows the comparison of GA, and Table 3 illustrates the similarities of TA activation during single and dual tasks, revealing no significant differences in activity between the different balance activities.

Table 4 demonstrates the comparability of neuromuscular coordination patterns between TA and GA during single tasks. The results indicated similar findings in coordination between TA and GA. However, the GA took longer to activate (TP: $0.70 \pm 0.53)$ than the TA (TP: $0.44 \pm 0.29)$ during EC ( $p=0.01)$, and GA had a prolonged activation (decay) compared with TA during ECHUD ( $p=0.005)$. Table 5 depicts a comparison of coordination patterns between TA and GA during dual tasks, showing similar activation outcomes of the muscles, with no significant differences during the varying balance conditions.

Table 2. Comparisons of gastrocnemius during single and dual tasks variables

\begin{tabular}{|c|c|c|c|c|c|}
\hline$n=44$ & Dual task (mean) & Dual task $(S D)$ & Single task (mean) & Single task $(S D)$ & $p$ \\
\hline Onset EO & 9.68 & 5.26 & 7.78 & 5.57 & 0.13 \\
\hline TP EO & 0.54 & 0.41 & 0.49 & 0.44 & 0.66 \\
\hline Decay EO & 0.54 & 0.42 & 0.55 & 0.45 & 0.91 \\
\hline Duration EO & 1.07 & 0.67 & 1.04 & 0.74 & 0.84 \\
\hline Onset EC & 7.33 & 4.27 & 8.31 & 4.52 & 0.33 \\
\hline TP EC & 0.51 & 0.53 & 0.69 & 0.53 & 0.15 \\
\hline Decay EC & 0.63 & 0.59 & 0.61 & 0.37 & 0.87 \\
\hline Duration EC & 1.14 & 0.94 & 1.30 & 0.75 & 0.42 \\
\hline Onset EOHUD & 8.92 & 5.61 & 7.20 & 4.57 & 0.14 \\
\hline TP EOHUD & 0.57 & 0.56 & 0.51 & 0.40 & 0.57 \\
\hline Decay EOHUD & 0.75 & 0.88 & 0.61 & 0.39 & 0.37 \\
\hline Duration EOHUD & 1.32 & 1.22 & 1.12 & 0.66 & 0.37 \\
\hline Onset ECHUD & 8.49 & 5.64 & 6.94 & 4.86 & 0.20 \\
\hline TP ECHUD & 0.72 & 1.08 & 0.51 & 0.31 & 0.26 \\
\hline Decay ECHUD & 0.52 & 0.41 & 0.72 & 0.43 & 0.05 \\
\hline Duration ECHUD & 1.20 & 1.17 & 1.23 & 0.60 & 0.90 \\
\hline
\end{tabular}

Results of MANOVA performed comparing electromyographic variables. Statistical significance at $p<0.01$.

EO - eyes open, EC - eyes closed, EOHUD - eyes open with head nods, ECHUD - eyes closed with head nods, onset - time to muscle activation onset, TP - time to peak muscle activation, decay - decay of muscle contraction, duration - muscle contraction duration 


\section{HUMAN MOVEMENT}

M. Rosario, A. Jose, Balance musculature modification

Table 3. Comparisons of tibialis anterior during single and dual tasks variables

\begin{tabular}{|c|c|c|c|c|c|}
\hline$n=44$ & Dual task (mean) & Dual task $(S D)$ & Single task (mean) & Single task $(S D)$ & $p$ \\
\hline Onset EO & 9.60 & 4.4 & 8.08 & 4.00 & 0.12 \\
\hline TP EO & 0.50 & 0.59 & 0.54 & 0.68 & 0.67 \\
\hline Decay EO & 0.57 & 0.58 & 0.58 & 0.44 & 0.94 \\
\hline Duration EO & 1.05 & 1.10 & 1.10 & 1.00 & 0.77 \\
\hline Onset EC & 8.72 & 5.14 & 8.64 & 5.24 & 0.37 \\
\hline TP EC & 0.46 & 0.24 & 0.44 & 0.29 & 0.62 \\
\hline Decay EC & 0.52 & 0.35 & 0.59 & 0.33 & 0.33 \\
\hline Duration EC & 0.98 & 0.48 & 1.03 & 0.47 & 0.15 \\
\hline Onset EOHUD & 9.56 & 4.74 & 8.32 & 4.28 & 0.23 \\
\hline TP EOHUD & 0.58 & 0.31 & 0.48 & 0.28 & 0.13 \\
\hline Decay EOHUD & 0.62 & 0.54 & 0.47 & 0.29 & 0.12 \\
\hline Duration EOHUD & 1.20 & 0.68 & 0.95 & 0.43 & 0.05 \\
\hline Onset ECHUD & 8.25 & 5.60 & 8.02 & 5.78 & 0.86 \\
\hline TP ECHUD & 0.45 & 0.29 & 0.47 & 0.26 & 0.78 \\
\hline Decay ECHUD & 0.56 & 0.33 & 0.49 & 0.22 & 0.27 \\
\hline Duration ECHUD & 1.00 & 0.45 & 0.95 & 0.35 & 0.56 \\
\hline
\end{tabular}

Results of MANOVA performed comparing electromyographic variables. Statistical significance at $p<0.01$. EO - eyes open, EC - eyes closed, EOHUD - eyes open with head nods, ECHUD - eyes closed with head nods, onset - time to muscle activation onset, TP - time to peak muscle activation, decay - decay of muscle contraction, duration - muscle contraction duration

Table 4. Comparisons of tibialis anterior and gastrocnemius during single tasks variables

\begin{tabular}{|c|c|c|c|c|c|}
\hline$n=44$ & GA (mean) & GA $(S D)$ & TA (mean) & $\mathrm{TA}(S D)$ & $p$ \\
\hline Onset EO & 7.78 & 5.57 & 8.08 & 3.96 & 0.79 \\
\hline TP EO & 0.49 & 0.44 & 0.54 & 0.68 & 0.72 \\
\hline Decay EO & 0.57 & 0.44 & 0.58 & 0.44 & 0.70 \\
\hline Duration EO & 1.04 & 0.74 & 1.13 & 1.00 & 0.67 \\
\hline Onset EC & 8.31 & 4.52 & 8.64 & 5.24 & 0.77 \\
\hline TP EC & 0.70 & 0.53 & 0.44 & 0.29 & $0.01 *$ \\
\hline Decay EC & 0.61 & 0.37 & 0.59 & 0.33 & 0.75 \\
\hline Duration EC & 1.30 & 0.75 & 1.03 & 0.47 & 0.06 \\
\hline Onset EOHUD & 7.20 & 4.57 & 8.32 & 4.28 & 0.27 \\
\hline TP EOHUD & 0.51 & 0.40 & 0.48 & 0.28 & 0.72 \\
\hline Decay EOHUD & 0.61 & 0.39 & 0.47 & 0.29 & 0.07 \\
\hline Duration EOHUD & 1.11 & 0.66 & 0.95 & 0.43 & 0.19 \\
\hline Onset ECHUD & 6.94 & 4.86 & 8.02 & 5.78 & 0.37 \\
\hline TP ECHUD & 0.51 & 0.31 & 0.47 & 0.26 & 0.44 \\
\hline Decay ECHUD & 0.72 & 0.43 & 0.49 & 0.22 & $0.005^{*}$ \\
\hline Duration ECHUD & 1.23 & 0.60 & 0.95 & 0.35 & 0.05 \\
\hline
\end{tabular}

Results of MANOVA performed comparing electromyographic variables. Statistical significance at $p<0.01$

(* statistically significant values).

GA - gastrocnemius, TA - tibialis anterior, EO - eyes open, EC - eyes closed, EOHUD - eyes open with head nods, ECHUD - eyes closed with head nods, onset - time to muscle activation onset, TP - time to peak muscle activation, decay - decay of muscle contraction, duration - muscle contraction duration 
Table 5. Comparisons of tibialis anterior and gastrocnemius during dual tasks variables

\begin{tabular}{lccccc}
\hline$n=44$ & GA (mean) & GA $(S D)$ & TA (mean) & TA (SD) & $p$ \\
\hline Onset EOC & 9.68 & 5.26 & 9.60 & 4.44 & 0.94 \\
TP EOC & 0.54 & 0.41 & 0.48 & 0.59 & 0.61 \\
Decay EOC & 0.54 & 0.42 & 0.58 & 0.58 & 0.72 \\
Duration EOC & 1.07 & 0.67 & 1.05 & 0.51 & 0.93 \\
Onset ECC & 7.33 & 4.27 & 8.72 & 5.14 & 0.20 \\
TP ECC & 0.51 & 0.53 & 0.46 & 0.24 & 0.59 \\
Decay ECC & 0.63 & 0.59 & 0.52 & 0.35 & 0.31 \\
Duration ECC & 1.14 & 0.94 & 0.98 & 0.48 & 0.33 \\
Onset EOCHUD & 8.92 & 5.61 & 9.56 & 4.74 & 0.59 \\
TP EOCHUD & 0.57 & 0.56 & 0.58 & 0.31 & 0.91 \\
Decay EOCHUD & 0.75 & 0.88 & 0.62 & 0.54 & 0.45 \\
Duration EOCHUD & 1.32 & 1.22 & 1.20 & 0.68 & 0.61 \\
Onset ECCHUD & 8.49 & 5.64 & 8.25 & 5.60 & 0.85 \\
TP ECCHUD & 0.72 & 1.08 & 0.45 & 0.29 & 0.13 \\
Decay ECCHUD & 0.52 & 0.41 & 0.56 & 0.33 & 0.64 \\
Duration ECCHUD & 1.20 & 1.17 & 1.01 & 0.45 & 0.33 \\
\hline
\end{tabular}

Results of MANOVA performed comparing electromyographic variables. Statistical significance at $p<0.01$.

GA - gastrocnemius, TA - tibialis anterior, EOC - eyes open cognitive, ECC - eyes closed cognitive,

EOCHUD - eyes open with head nods during cognitive task, ECCHUD - eyes closed with head nods

during cognitive task, onset - time to muscle activation onset, TP - time to peak muscle activation,

decay - decay of muscle contraction, duration - muscle contraction duration

The neuromuscular timing of the TA was compared during single and dual tasks (Table 6), and similar comparisons were made for the neuromuscular timing of the GA (Table 7). Outcomes indicated comparable neuromuscular recruitment patterns for both the TA and GA with no significant distinctions during the various tasks.

\section{Discussion}

This study intended to distinguish whether TA and GA EMG activity was comparable during single and dual tasks when the sensory systems were challenged in young adults. Therefore, the study tried to answer if a single and dual-cognitive balance task in assortment with several challenging procedures elicited a specific neuromuscular pattern in lower limb musculature in young healthy adults. We hypothesized that the neuromuscular timing activity of TA and GA would be indiscernible during the different sensory conditions owing to healthy sensory systems and efferent motor reactions in young adults. The outcomes of this inquiry display, for the most part, no significant differences in TA and GA EMG activity during the different tasks, irrespective of what sensory systems were challenged. Therefore, we accept our hypothesis.
The 8 different balance tests in our investigation were all performed on a foam surface to alter the proprioceptive input. Visual input is cancelled during eyes closed tasks; thus, sensory information must be redirected to the other sensory systems to preserve balance. The summation of head movements challenges the vestibular system. When all sensory systems are disputed coincidentally, preserving postural stability becomes, to a greater extent, strenuous.

The corresponding changes in TA and GA EMG activity between tasks indicate that our participants have intact sensory and neuromuscular systems responsible for maintaining balance. Giulio et al. [13] mention that managing standing balance is still possible solely from sensory information coming from the ankle muscles, even when the other sensory systems are unavailable. Allocating attentional resources effectively during various balance conditions is critical for postural stability; nonetheless, adding a cognitive task could increase interference in sensory processing. A similar study revealed that the onset of muscle activity for GA and TA was unaltered during dual task (math and balance tasks). Still, the amplitude of muscle activity decreased in both young adults and older adults, though changes were more apparent in older adults [14]. Nevertheless, in the current study, only 


\section{HUMAN MOVEMENT}

M. Rosario, A. Jose, Balance musculature modification

Table 6. Comparisons of neuromuscular variables of tibialis anterior, onset, TP, duration, and decay during single and dual tasks on a foam surface

\begin{tabular}{|c|c|c|c|}
\hline Single task $(n=44)$ & Mean and $S D$ & Mean and $S D$ & $p$ \\
\hline Onset & EO: $8.08 \pm 3.96$ & $\begin{array}{l}\text { EC: } 8.64 \pm 5.24 \\
\text { EOHUD: } 8.32 \pm 4.28 \\
\text { ECHUD: } 8.02 \pm 5.78 \\
\text { EOC: } 9.60 \pm 4.44 \\
\text { ECC: } 8.72 \pm 5.14 \\
\text { EOCHUD: } 9.56 \pm 4.74 \\
\text { ECCHUD: } 8.25 \pm 5.60\end{array}$ & $\begin{array}{l}>0.99 \\
>0.99 \\
>0.99 \\
>0.99 \\
>0.99 \\
>0.99 \\
>0.99\end{array}$ \\
\hline $\mathrm{TP}$ & EO: $0.54 \pm 0.68$ & $\begin{array}{l}\text { EC: } 0.44 \pm 0.29 \\
\text { EOHUD: } 0.48 \pm 0.28 \\
\text { ECHUD: } 0.47 \pm 0.26 \\
\text { EOC: } 0.48 \pm 0.59 \\
\text { ECC: } 0.46 \pm 0.24 \\
\text { EOCHUD: } 0.58 \pm 0.31 \\
\text { ECCHUD: } 0.45 \pm 0.29\end{array}$ & $\begin{array}{l}>0.99 \\
>0.99 \\
>0.99 \\
>0.99 \\
>0.99 \\
>0.99 \\
>0.99\end{array}$ \\
\hline Duration & EO: $1.13 \pm 1.00$ & $\begin{array}{l}\text { EC: } 1.03 \pm 0.47 \\
\text { EOHUD: } 0.95 \pm 0.43 \\
\text { ECHUD: } 0.95 \pm 0.35 \\
\text { EOC: } 1.06 \pm 1.11 \\
\text { ECC: } 0.98 \pm 0.48 \\
\text { EOCHUD: } 1.20 \pm 0.68 \\
\text { ECCHUD: } 1.00 \pm 0.45\end{array}$ & $\begin{array}{l}>0.99 \\
>0.99 \\
>0.99 \\
>0.99 \\
>0.99 \\
>0.99 \\
>0.99\end{array}$ \\
\hline Decay & EO: $0.58 \pm 0.44$ & $\begin{array}{l}\text { EC: } 0.59 \pm 0.33 \\
\text { EOHUD: } 0.47 \pm 0.29 \\
\text { ECHUD: } 0.49 \pm 0.22 \\
\text { EOC: } 0.58 \pm 0.58 \\
\text { ECC: } 0.52 \pm 0.35 \\
\text { EOCHUD: } 0.62 \pm 0.54 \\
\text { ECCHUD: } 0.56 \pm 0.33\end{array}$ & $\begin{array}{l}>0.99 \\
>0.99 \\
>0.99 \\
>0.99 \\
>0.99 \\
>0.99 \\
>0.99\end{array}$ \\
\hline
\end{tabular}

Results of repeated measures ANOVA performed comparing variables.

Statistical significance at $p<0.01$.

Onset - time to muscle activation onset, TP - time to peak muscle activation,

duration - muscle contraction duration, decay - decay of muscle contraction, EO - eyes open, EC - eyes closed, EOHUD - eyes open with head nods, ECHUD - eyes closed with head nods, EOC - eyes open cognitive, ECC - eyes closed cognitive, EOCHUD - eyes open with head nods during cognitive task, ECCHUD - eyes closed with head nods during cognitive task

minimal changes in neuromuscular timing activity were found during dual-cognitive tasks in our participants.

Additional studies have shown adequate postural control during dual tasks compared with single tasks even when the sensory input is altered $[15,16]$. Wulf et al. [17] elucidate how externally focused instructions (effects of the movement) promote automaticity in motor control compared with internally focused instructions (how movement is produced) during complex balance tasks. We instructed our participants to preserve balance during the different tasks instead of focusing on what part of their body is moving, which would evoke an external focus of attention. Seeing no substantial changes in the variables of interest during the different tasks implies that our participants had an external focus of attention, which allowed automatic postural control even when a cognitive task was added to the balance tests. Similarly, another study reveals that postural control during cognitive tasks is a result of automatic postural control rather than stiffening of the ankle muscles (increased activation of muscles) [18]. Future studies should consider exploring whether there is a correlation between neuromuscular activity and temporal-spatial parameters during various balance activities. Additionally, pro- 
Table 7. Comparisons of neuromuscular variables of gastrocnemius, onset, TP, duration, and decay during single and dual tasks on a foam surface

\begin{tabular}{|c|c|c|c|}
\hline Single task $(n=44)$ & Mean and $S D$ & Mean and $S D$ & $p$ \\
\hline Onset & EO: $7.78 \pm 5.57$ & $\begin{array}{l}\text { EC: } 8.31 \pm 4.52 \\
\text { EOHUD: } 7.20 \pm 4.57 \\
\text { ECHUD: } 6.94 \pm 4.86 \\
\text { EOC: } 9.68 \pm 5.26 \\
\text { ECC: } 7.33 \pm 4.27 \\
\text { EOCHUD: } 8.92 \pm 5.61 \\
\text { ECCHUD: } 8.50 \pm 5.64\end{array}$ & $\begin{aligned}> & 0.99 \\
> & 0.99 \\
> & 0.99 \\
> & 0.99 \\
> & 0.99 \\
> & 0.99 \\
& 0.34\end{aligned}$ \\
\hline $\mathrm{TP}$ & EO: $0.49 \pm 0.44$ & $\begin{array}{l}\text { EC: } 0.69 \pm 0.53 \\
\text { EOHUD: } 0.51 \pm 0.40 \\
\text { ECHUD: } 0.51 \pm 0.31 \\
\text { EOC: } 0.54 \pm 0.41 \\
\text { ECC: } 0.51 \pm 0.53 \\
\text { EOCHUD: } 0.57 \pm 0.56 \\
\text { ECCHUD: } 0.72 \pm 1.08\end{array}$ & $\begin{array}{l}>0.99 \\
>0.99 \\
>0.99 \\
>0.99 \\
>0.99 \\
>0.99 \\
>0.99\end{array}$ \\
\hline Duration & EO: $1.04 \pm 0.74$ & $\begin{array}{l}\text { EC: } 1.30 \pm 0.75 \\
\text { EOHUD: } 1.12 \pm 0.66 \\
\text { ECHUD: } 1.23 \pm 0.60 \\
\text { EOC: } 1.07 \pm 0.67 \\
\text { ECC: } 1.14 \pm 0.94 \\
\text { EOCHUD: } 1.32 \pm 1.22 \\
\text { ECCHUD: } 1.20 \pm 1.17\end{array}$ & $\begin{array}{l}>0.99 \\
>0.99 \\
>0.99 \\
>0.99 \\
>0.99 \\
>0.99 \\
>0.99\end{array}$ \\
\hline Decay & EO: $0.55 \pm 0.45$ & $\begin{array}{l}\text { EC: } 0.61 \pm 0.37 \\
\text { EOHUD: } 0.61 \pm 0.39 \\
\text { ECHUD: } 0.72 \pm 0.43 \\
\text { EOC: } 0.54 \pm 0.42 \\
\text { ECC: } 0.63 \pm 0.59 \\
\text { EOCHUD: } 0.75 \pm 0.88 \\
\text { ECCHUD: } 0.52 \pm 0.41\end{array}$ & $\begin{array}{l}>0.99 \\
>0.99 \\
>0.99 \\
>0.99 \\
>0.99 \\
>0.99 \\
>0.99\end{array}$ \\
\hline
\end{tabular}

Results of repeated measures ANOVA performed comparing variables.

Statistical significance at $p<0.01$.

Onset - time to muscle activation onset, TP - time to peak muscle activation,

duration - muscle contraction duration, decay - decay of muscle contraction, EO - eyes open, EC - eyes closed, EOHUD - eyes open with head nods, ECHUD - eyes closed with head nods, EOC - eyes open cognitive, ECC - eyes closed cognitive, EOCHUD - eyes open with head nods during cognitive task, ECCHUD - eyes closed with head nods during cognitive task

spective inquiries should investigate the impact of dualmotor tasks on neuromuscular activation.

The neuromuscular coordination patterns between the agonist and antagonist muscles should be considered to further comprehend balance control at the ankle. Muscles are unidirectional force generators, which means muscles are unable to simultaneously have a push and pull mechanism [3]. Thus, at least 2 muscles (agonist and antagonist) are needed to control the ankle joint. Previous EMG studies have mentioned that the GA acts as an agonist, and the TA acts as an antagonist at the ankle joint during a quiet stance [13]. Based on the results of the variables of interest, the consistent neuromuscular coordination patterns between the 2 muscles imply that there were no significant fluctuations in anterior-posterior sway due to simultaneous coactivation of GA and TA. This presumption further supports our hypothesis that young adults can maintain static balance owing to efficient coordination between afferent inputs and efferent outputs. Nonetheless, the first EC task had a prolonged average TP of GA, which means that sway was going posterior first, creating a dorsiflexion force. This posterior sway could be because participants had visual dependence for balance. Still, they were able to adapt to the remaining balance tests since no substantial 
changes in activation patterns were demonstrated. Previous studies have identified increased anterior-posterior sway when eyes are closed [19]. The prolonged activation time of GA compared with TA during ECHUD tasks is plausible because if there is increased anterior-posterior sway when eyes are closed, then a consistent plantar flexion torque by the GA is essential to prevent the body from falling forward.

In addition to the previous findings, the neuromuscular recruitment patterns were parallel during single and dual tasks for both GA and TA muscles. These comparable results purport that neither the complexity of the balance test nor the addition of a cognitive component creates significant interference in central processing or muscle activity in young adults. These outcomes also indicate that any deviations to such a pattern could be an alteration to normal neuromuscular recruitment.

Finally, the reasoning behind our research findings could be interpreted and explained by motor control theories. Wulf et al. [20] deduce that during a dualcognitive task, postural control becomes automatic. At the same time, the attentional resources are redirected to the cognitive task, enabling one to perform the cognitive task without losing balance. Our participants conceivably were able to have an automatic motor response to balance tests while effectively regulating the modulation of attentional resources for the dualcognitive task. Postural stability also involves muscle synergy, which is the activation of a group of muscles, and it plays a role in mitigating excessive demands to central processing [1]. For example, during quiet stance, the synergistic activation of hamstrings and paraspinal musculature keeps the hips and knees extended, and GA prevents the forward motion of the body by creating a plantar flexion torque [1]. The corresponding results of our study could be due to postural synergistic muscles compensating for any postural imbalance, requiring less activation of the TA and GA during demanding tasks.

One possible limitation of our study is the variability in the placement of the electrodes. Though the Sacco and Kasman protocol for electrode placement was followed and the muscle belly for TA and GA were located for EMG sensor placement, the research assistants did not measure the exact distance of electrode placement site from the anatomical landmarks, which possibly could have altered EMG activity. Prospective studies should establish a standardized protocol for measuring the distance between the electrode placement site and the anatomical landmarks. Additionally, the accuracy and the response time of the mathematical calculations pertaining to the dual- cognitive task were not assessed. Rather the highest peak of muscle activity in each task was identified and compared in this study, similar to Rosario et al. [21] research. One final note is essential to discern the standard modification or adaptation of muscle activation to recognize when injury or pathologies endangered the regular muscle-balance interaction and could perturb equilibrium, increasing the fall risk. According to the methodology approach assumed by Bello et al. [22], future studies should focus on different types of muscle contraction, concentric and eccentric, when investigating the relationship of dual balance activities. This information can be potentially useful to understand the mechanism of injury and for an effective rehabilitation period. Additionally, prospective inquiries should focus on everyday surfaces such as sand, grass, and gravel, for instance, to explain the role and modification of lower extremities muscles under challenging yet normal surfaces during dual-tasks standing balance.

\section{Conclusions}

This study proffers that similar neuromuscular activity of the TA and GA during complex postural tasks is a result of intact sensory and motor systems in young healthy adults. Future studies should examine the neuromuscular activity of other postural muscles, such as the gluteus, hamstrings, and paraspinal musculature to interpret postural muscle activity holistically during different balance tests. Future studies can also expand on comparing EMG response between neuromuscular populations and healthy populations. Moreover, research utilizing electroencephalography along with EMG can help recognize whether there are changes in brain activity during single and dual tasks. Lastly, this study solely pertains to EMG activity during single and cognitive tasks; further research needs to identify whether EMG activity is altered when dual-motor tasks are introduced.

\section{Disclosure statement}

No author has any financial interest or received any financial benefit from this research.

\section{Conflict of interest}

The authors state no conflict of interest.

\section{References}

1. Shumway-Cook A, Woollacott MH. Motor control: theory and practical applications, $2^{\text {nd }}$ ed. Philadelphia: Lippincott Williams \& Wilkins; 2001. 
2. Base of support. Physiopedia; n.d. Available from: https://www.physio-pedia.com/Base_of_Support.

3. Latash ML. Fundamentals of motor control. Elsevier; 2012.

4. Goodman CC, Snyder TEK. Differential diagnosis for physical therapists, $5^{\text {th }}$ ed. Elsevier; 2013.

5. Gurvich C, Maller JJ, Lithgow B, Haghgooie S, Kulkarni J. Vestibular insights into cognition and psychiatry. Brain Res. 2013;1537:244-259; doi: 10.1016/j. brainres.2013.08.058.

6. Sensory processing FAQ. Child Mind Institute; n.d. Available from: https://childmind.org/article/sensoryprocessing-faq/.

7. Rosario MG, López L, Méndez M, Ababneh AF, Gonzalez-Sola M. Proprioception and vestibular alterations affect postural control in children with mild autism: a pilot study [version 1; peer review: 1 approved, 1 approved with reservations]. F1000Research. 2018;7:305; doi: 10.12688/f1000research.14179.1.

8. Horlings CGC, van Engelen BGM, Allum JHJ, Bloem BR. A weak balance: the contribution of muscle weakness to postural instability and falls. Nat Clin Pract Neurol. 2008;4(9):504-515; doi: 10.1038/ncpneuro0886.

9. Hsu W-L, Scholz JP, Schöner G, Jeka JJ, Kiemel T. Control and estimation of posture during quiet stance depends on multijoint coordination. J Neurophysiol. 2007;97(4):3024-3035; doi: 10.1152/jn.01142.2006.

10. Cameron MH, Horak FB, Herndon RR, Bourdette D. Imbalance in multiple sclerosis: a result of slowed spinal somatosensory conduction. Somatosens Mot Res. 2008;25(2):113-122; doi:10.1080/08990220802131127.

11. Tsuchida W, Nakagawa K, Kawahara Y, Yuge L. Influence of dual-task performance on muscle and brain activity. Int J Rehabil Res. 2013;36(2):127-133; doi: 10.1097/MRR.0b013e32835acfb8.

12. Little CE, Woollacott M. EEG measures reveal dualtask interference in postural performance in young adults. Exp Brain Res. 2015;233(1):27-37; doi: 10.1007/ s00221-014-4111-x.

13. Giulio ID, Maganaris CN, Baltzopoulous V, Loram ID. The proprioceptive and agonist roles of gastrocnemius, soleus and tibialis anterior muscles in maintaining human upright posture. J Physiol. 2009;587(Pt 10):2399_ 2416; doi: 10.1113/jphysiol.2009.168690.

14. Rankin JK, Woollacott MH, Shumway-Cook A, Brown LA. Cognitive influence on postural stability: a neuromuscular analysis in young and older adults. J Gerontol A Biol Sci Med Sci. 2000;55(3):M112-M119; doi: 10.1093/gerona/55.3.m112.

15. Resch JE, May B, Tomporowski PD, Ferrara MS. Balance performance with a cognitive task: a continuation of the dual-task testing paradigm. J Athl Train. 2011; 46(2):170-175; doi: 10.4085/1062-6050-46.2.170.

16. Kiss R, Brueckner D, Muehlbauer T. Effects of single compared to dual task practice on learning a dynamic balance task in young adults. Front Psychol. 2018;9: 311; doi: 10.3389/fpsyg.2018.00311.
17. Wulf G, Höß M, Prinz W. Instructions for motor learning: differential effects of internal versus external focus of attention. J Mot Behav. 1998;30(2):169179; doi: 10.1080/00222899809601334.

18. Richer N, Saunders D, Polskaia N, Lajoie Y. The effects of attentional focus and cognitive tasks on postural sway may be the result of automaticity. Gait Posture. 2017;54:45-49; doi: 10.1016/j.gaitpost.2017.02.022.

19. Barbieri FA, Penedo T, Simieli L, Barbieri RA, Zagatoo AM, van Diëen JH, et al. Effects of ankle muscle fatigue and visual behavior on postural sway in young adults. Front Physiol. 2019;10:643; doi: 10.3389/ fphys.2019.00643.

20. Wulf G, McNevin N, Shea CH. The automaticity of complex motor skill learning as a function of attentional focus. Q J Exp Psychol A. 2001;54(4):1143-1154; doi: 10.1080/713756012.

21. Rosario MG, Jamison L, Hyder A. The influence of energy drinks on lower limb neuromuscular timing and postural sway in healthy young adults. J Pub Health Issue Pract. 2020;4(1):163; doi: 10.33790/ jphip1100 163.

22. Bello FD, Aedo-Muñoz E, Moreira DG, Brito CJ, Miarka B, Cabello EN. Beach and indoor volleyball athletes present similar lower limb muscle activation during a countermovement jump. Hum Mov. 2020;21(2):42-50; doi: $10.5114 / \mathrm{hm} .2020 .89913$. 